\title{
A survey of current practices by the British Oculoplastic Surgery Society (BOPSS) and recommendations for delivering a sustainable multidisciplinary approach to thyroid eye disease in the United Kingdom
}

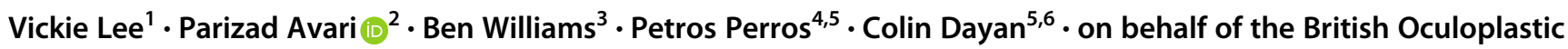 \\ Surgery Society (BOPSS) and TEAMeD
}

Received: 26 June 2019 / Revised: 27 September 2019 / Accepted: 14 October 2019 / Published online: 13 December 2019

(c) The Author(s), 2019. This article is published with open access

\begin{abstract}
Background The Royal College of Physicians (RCP) and Thyroid Eye Disease Amsterdam Declaration Implementation Group (TEAMeD-5) have the common goal of improving access to high quality care for thyroid eye disease (TED). The TEAMeD-5 programme recommends all patients with moderate-to-severe TED should have access to multidisciplinary clinics (MDT) with combined Ophthalmology and Endocrinology expertise.

Methods The British Oculoplastic Surgery Society represents oculoplastic surgeons who usually lead TED care in the UK. A two-stage survey of the membership was conducted to ascertain current practice of existing resources.

Results Seventy percent (45/65) of respondents in Survey 1 were aware of current RCP guidance, but only 49\% (22/45) rated it as a good means of improving access to comprehensive TED service. Sixty percent (39/65) of respondents are working in a multidisciplinary TED clinic with co-location of ophthalmologists and endocrinologists. Care for TED appears not to be provided in a multidisciplinary context in up to $31 \%$ (20/65). Thirty five (54\%) of the respondents rated their relationship with endocrinology colleagues as good. Best practice guidelines recommend routine quality of life assessments but only 6/28 (21\%) of respondents use this modality in current practice. Six percent (4/65) of areas appear not to be using intravenous steroids. In many areas $(25 \%, 16 / 65)$, second-line immunosuppression is provided in a different trust and in $8 \%$ (5/65), it appears not to be used at all.

Conclusion This survey is a 'snapshot' of current TED management in the UK and findings suggest scope for improvement. We recommend a framework for more robust collaboration across specialties and propose standards endorsed by multidisciplinary stakeholder societies.
\end{abstract}

Supplementary information The online version of this article (https:// doi.org/10.1038/s41433-019-0664-z) contains supplementary material, which is available to authorized users.

Vickie Lee

vickie.lee@nhs.net

1 Department of Ophthalmology, Imperial College London NHS Trust, London, UK

2 Department of Diabetes and Endocrinology, Imperial College London, London, UK

3 Department of Website Support, MediSites, Bournemouth, UK

\section{Introduction}

Multidisciplinary clinics for complex disease have become the gold standard of care in recent years. This is based on the premise that care given by a group of clinical professionals is more comprehensive than that provided by a single individual, and that specialist skills and knowledge

4 Department of Endocrinology, Newcastle Upon Tyne Hospitals NHS Foundation Trust, Newcastle Upon Tyne, UK

5 TEAMed Thyroid Eye Disease Amsterdam Declaration Implementation Group, Cardiff, UK

6 Thyroid Research Group, Cardiff University School of Medicine, Cardiff, UK 
are used more appropriately avoiding duplication and gaps in care.

Previous studies have reported age-adjusted annual incidence rates of $42 /$ million for thyroid eye disease (TED) of all severity (10/million for moderate-to-severe disease) [1]. The most accurate estimate of prevalence of all cases of TED in Europe is $0.1 \%$ [2]. The burden of disease can have significant impact on physical and psychosocial wellbeing, may be disfiguring and occasionally sight threatening [3, 4]. There is now robust evidence to show the severity of residual disease can be substantially decreased by timely specialist intervention [5]. The Amsterdam Declaration [6] led by the European Group on Graves' Orbitopathy (EUGOGO), and signed by over 80 professional and patient-led organisations in 2009, aimed to improve outcomes for patients with TED. Presentation of TED [7] and subsequent studies show multidisciplinary team (MDT) clinics improve access to care. Its recommendations include support for existing centres of excellence in management of this condition, the creation of new centres of excellence in localities where they are lacking and to establish audit and monitoring mechanisms of quality assurance of provision of care to people with TED. The 2008 consensus statement from EUGOGO [8] and the 2016 European Thyroid Association Guidelines on Graves' Orbitopathy [9] recommended joint multidisciplinary care between specialties (ophthalmology and endocrinology) for all cases of moderate or severe TED. Several studies from the UK and other European countries have highlighted delays in the diagnosis of TED and referral to specialist centres and that many patients with moderate or severe disease were not managed in appropriately skilled specialist centres [7, 10-13].

In the UK, Thyroid Eye Disease Amsterdam Declaration Implementation Group (TEAMeD: http://www.btf-thyroid. org/projects/teamed) was formed to implement the Amsterdam Declaration objectives and improve outcomes for patients with TED [7]. TEAMeD have subsequently conducted epidemiological surveys that have shown significant regional variation within the UK in care pathways and availability of specialist surgery [10, 11, 14]. Often significant delays have resulted in the active disease phase being missed, along with the window for disease modifying intervention [11]. In 2015, the Royal College of Physicians (RCP) (endorsed by Royal College of Ophthalmologists, BOPPS, British Thyroid Association and Society for Endocrinology) issued updated guidelines on management of patients with Graves' orbitopathy: initial assessment, management outside specialised centres and criteria for referral to specialist care [7] with additional guidance published subsequently by EUGOGO [9].

To implement these recommendations and raise awareness of prevention measures and early referral of TED amongst the endocrine community, the TEAMeD-5 programme was launched within the UK in 2017. Given that more than $80 \%$ of TED presents in patients with a known diagnosis of Graves' disease, TEAMeD-5 recommends [15] the following:

(1) accurate diagnosis of Graves' disease to identify patients at risk of TED (e.g. TSH receptor antibody testing);

(2) screening of all patients with Graves' disease for early symptoms and signs of TED;

(3) alerting individuals with Graves' disease to the early symptoms of TED using an awareness card;

(4) optimising measures to prevent and reduce the severity of TED by smoking reduction, early induction and maintenance of euthyroidism and avoidance of radioiodine in active TED;

(5) prompt referral of individuals with moderate or severe TED or TED affecting quality of life directly to a regional specialist multidisciplinary clinic with appropriate experience and expertise [16].

Prior to this initiative launch, the British Oculoplastic Surgery Society (BOPSS) liaised closely with TEAMeD and a two-part survey was designed to assess the readiness of ophthalmic services across the UK to comply with the recommendations.

\section{Methods}

This was the first full membership survey approved by the BOPSS Executive Committee and comprised of two sections. Survey 1 included questions (Supplementary Fig. 1) on the regional location of respondent, their awareness of the 2015 RCP guidance and perception of its utility, their relationship with their local endocrinology department and whether there were any screening arrangements for TED agreed locally. Further questions included the availability of a geographically accessible multidisciplinary combined thyroid eye clinic with Ophthalmologists and Endocrinologists, staffing provisions, treatment modalities available within their hospital trust and whether they used a particular treatment.

All respondents of Survey 1 were then invited to complete Survey 2, which included more detailed questions about their service configuration (Supplementary Fig. 1). The survey went 'live' on the BOPSS website in December 2016 with an email invitation to the full BOPSS membership consisting of post-CCT (Certificate of Completion of Training) ophthalmic surgeons with subspecialty expertise in oculoplastics. The preliminary findings of the survey were presented at the BOPSS London 2017 Annual Meeting by the authors to discuss various aspects of 


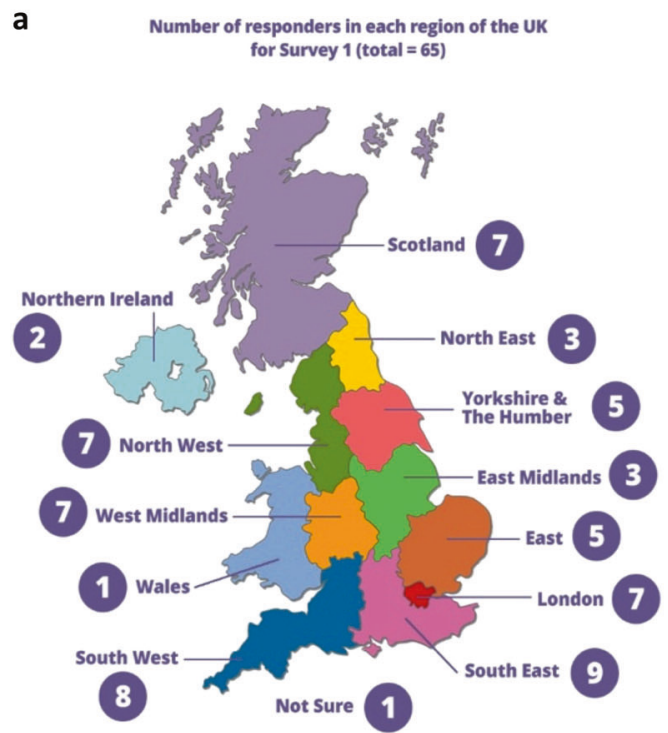

Fig. 1 Number of respondents from each region of the UK for a Survey 1 and b Survey 2 for whether patients with TED are managed within a multidisciplinary setting at minimum monthly intervals. For Survey 2, there were no respondents from Scotland or Wales. 'Specialist multidisciplinary (MDT) services' for TED management clinic

multidisciplinary working in collaboration with speakers across different regions of the UK showcasing their local services. The session terminated with an invitation to all full members who had not previously participated to complete the survey and a further email invitation was re-sent following the meeting. The survey closed in August 2017.

'Specialist multidisciplinary (MDT) services' for TED management clinic was defined as co-location of an endocrinologist with an ophthalmologist within the same clinic.

An online discussion of a group of full members was set up under the aegis of BOPSS with representatives from across the UK to discuss recommendations and regular progress reporting by author VL at the Executive Committee meetings. Data were presented at the full members' annual general meeting in 2018 for discussion and feedback. Draft versions of this paper were circulated to representatives of all stakeholder societies within TEAMeD and comments incorporated into subsequent versions

\section{Results}

The original survey was first sent out to all members $(n=$ 150) in December 2016 with a final survey reminder sent out to all BOPSS members $(n=158)$ in August 2017.

\section{Survey 1: general service configuration}

There were 65 respondents to Survey 1 (41\% of the BOPSS full membership) and 28 respondents (43\%) to Survey 2.

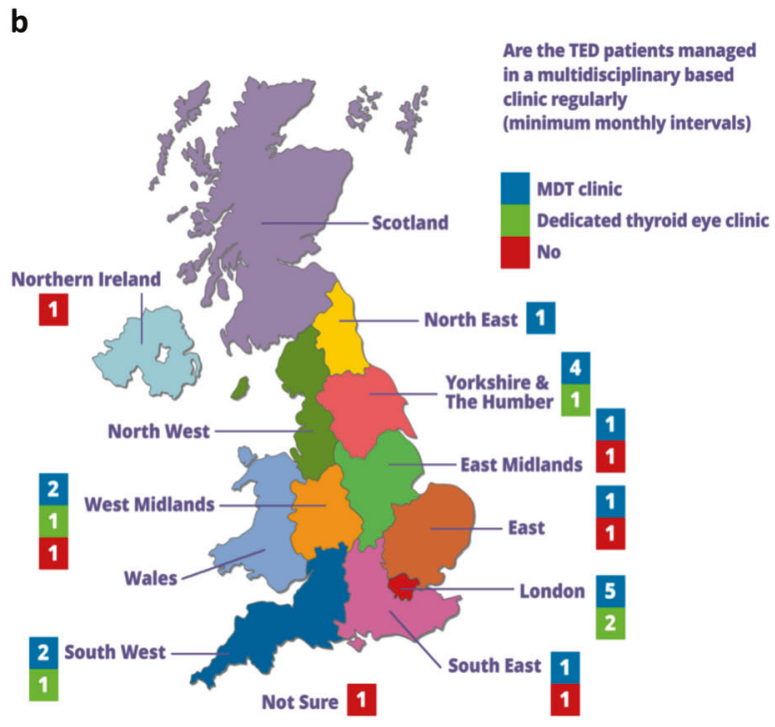

was defined as co-location of an endocrinologist with an ophthalmologist within the same clinic. A "dedicated thyroid eye clinic" was defined as a specialised ophthalmology clinic for thyroid eye patients only (does not include the presence of an endocrinologist)

Individuals across all regions in the UK participated for Survey 1 (Fig. 1a). Forty-five (69\%) respondents were aware of the 2015 guidance. Of these, 22/45 (49\%) considered that these as a good, 21/45 (47\%) as an average and $2 / 45(4 \%)$ as a poor guide to improving access of patients to comprehensive thyroid eye services.

Across the UK, 20/65 (29\%) respondents do not use specialist multidisciplinary services for TED management, with 39/65 (60\%) working in a dedicated multidisciplinary (MDT) thyroid eye clinic with co-location of endocrinology and ophthalmology and 6/65 (9\%) referring patients to another trust within a dedicated thyroid eye clinic setup (Fig. 2). Twenty respondents do not have an MDT thyroid eye clinic, respondents who do not use multidisciplinary services were located in seven out of nine geographical regions of the UK (Fig. 2).

Thirty-five respondents (54\%) rated their relationship with their local endocrinology department as good, 22/65 (34\%) average and 4/65 (6\%) poor and 4 (3\%) did not comment. Sixty-one (94\%) respondents used intravenous methyl prednisolone (IVMP) as part of TED management in their own trust. Two (3\%) respondents did not use IVMP and another two (3\%) referred patients to another trust for IVMP treatment. Forty four (68\%) offered second-line immunosuppression (when first-line therapy with intravenous steroids has failed/ ceased working) within their own trust. Forty (62\%) offered orbital decompression within their own trust, with over a third referred to another trust, and 3\% (2/65) responding that they did not offer orbital decompression to their TED patients (Supplementary Fig. 2). Sixty 

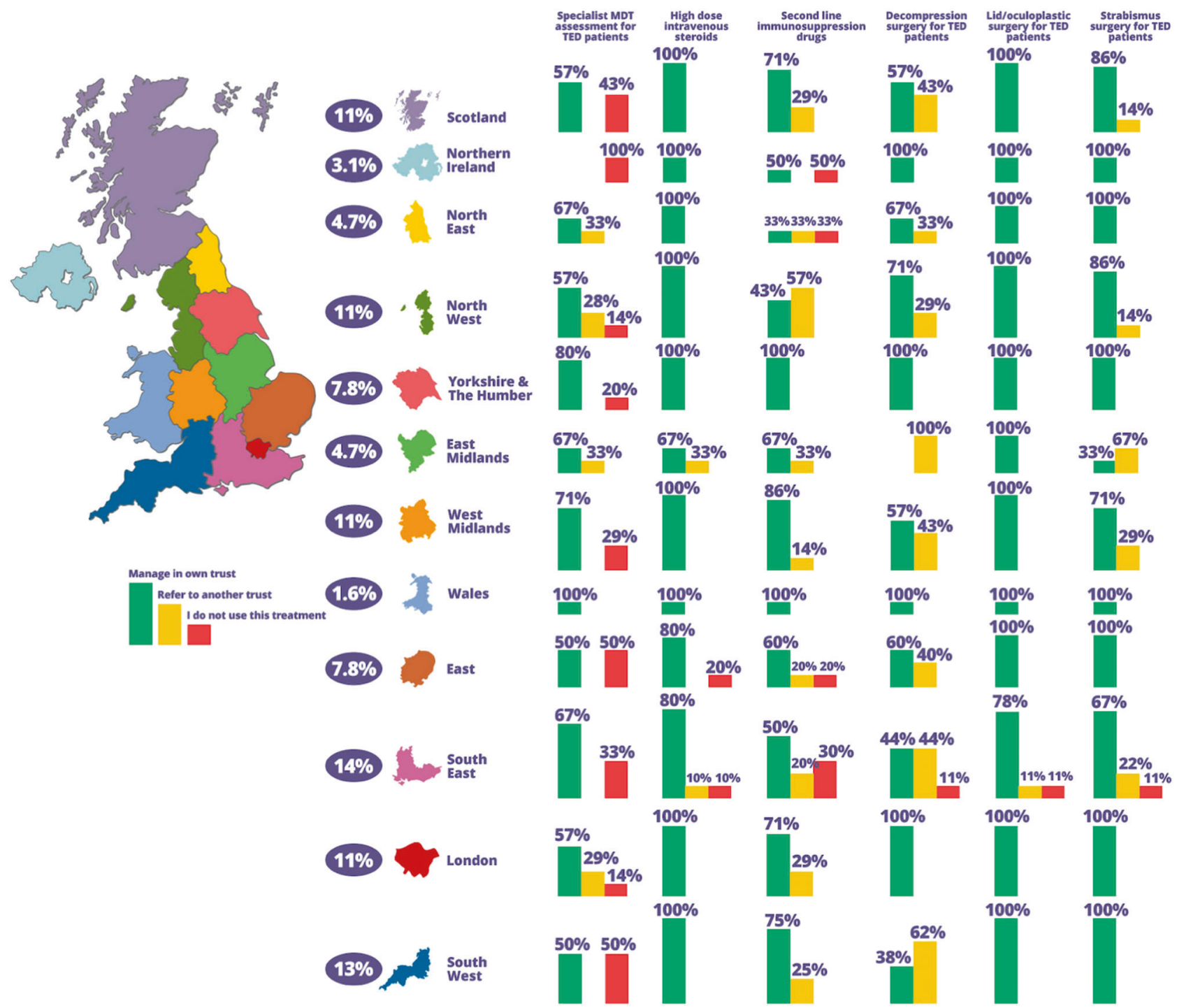

Fig. 2 Responses based on geographic location from Survey 1 on type of management provided within own clinical unit or referral to another

four (97\%) offered oculoplastic and fifty seven (88\%) strabismus surgery to TED patients within their own trust.

\section{Survey 2: detailed service configuration}

Twenty-eight (18\% of the membership) respondents completed Survey 2 (with no respondents from Wales or Scotland). Seventeen out of twenty eight (61\%) of the respondents stated that they treated patients within a MDT thyroid eye clinic with an endocrinologist present (Fig. 1b). Two-thirds (68\%) included orthoptic support, but only approximately a third (36\%) included other health professionals (including immunosuppression specialists). Fourteen out of twenty eight $(50 \%)$ responded that their local endocrine service did not routinely screen for TED. Of those who affirmed that screening did take place, two respondents stated that they did not know how their local endocrinologists screened for TED and the remainder reported that a variety of screening tools including Vancouver orbitopathy score and NOSPECS were used. Fifteen (54\%) reported that smoking cessation services were available within their own trust or locally, and the remainder said they did not know about local provision. Twentyfour $(85 \%)$ and $22(79 \%)$ individuals reported that thyroidectomy and radioiodine were carried out within their own trust.

Both MRI and CT scans (27/28) were widely used orbital imaging modalities. Twenty-three out of twenty-eight $(82 \%)$ respondents stated they routinely gave their patients written information regarding TED, but only $6 / 28$ (22\%) routinely collected patient reported outcomes and quality of life measurements. This more detailed survey 

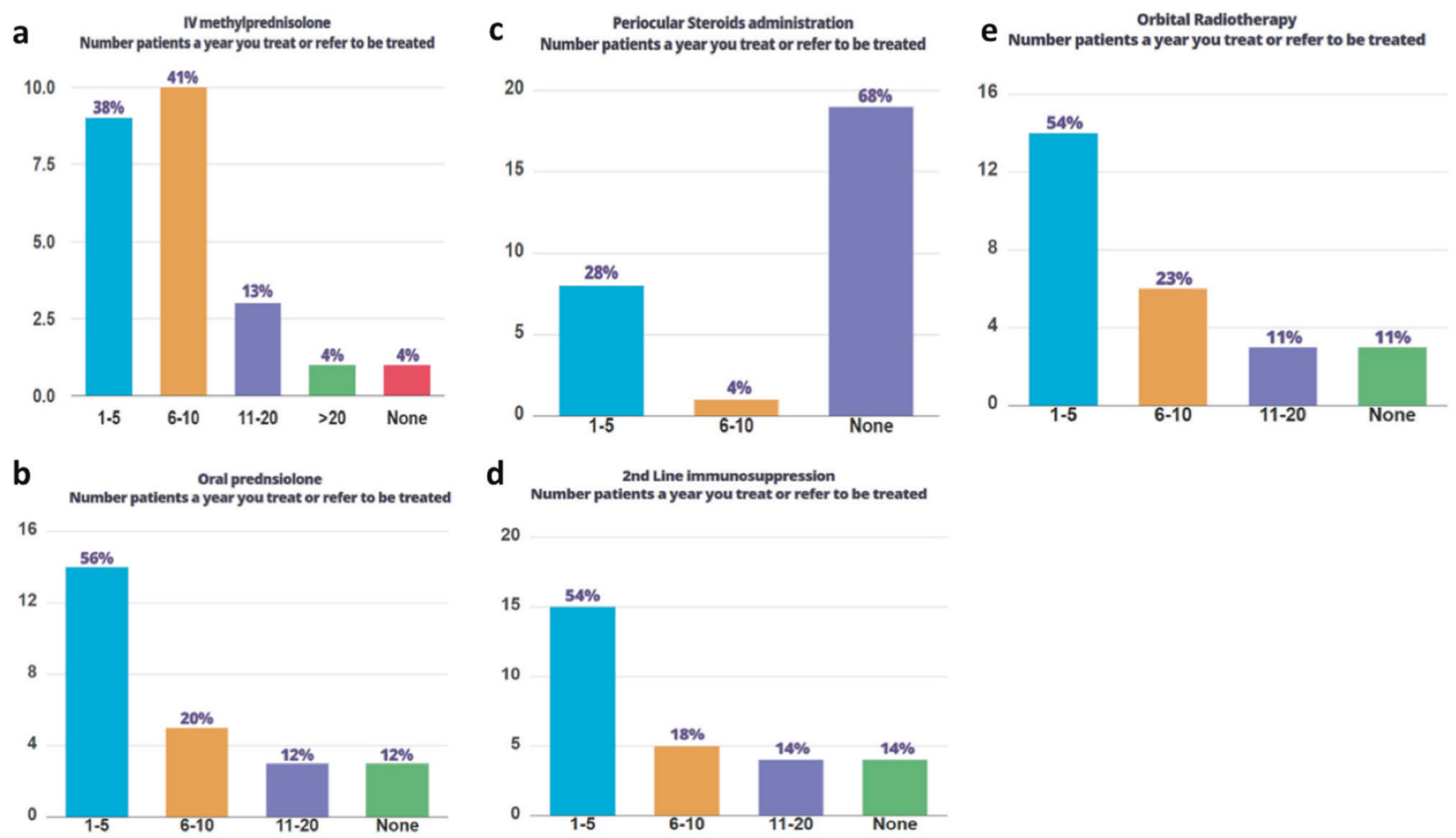

Fig. 3 Responses to Survey 2 on numbers treated with a intravenous, b oral, c peri-ocular steroid treatment, $\mathbf{d}$ second-line immunosuppression and e orbital radiotherapy

confirmed a large variation of treatment modalities and experience of using intravenous steroids, oral prednisone, periocular steroids and second-line immunosuppression (Fig. 3). Figure 3 reflects the number of patients treated/ referred per year by each respondent, according to treatment modality. Twenty seven (96\%) used intravenous steroids of which 16 were administered by the eye department (the remainder by other physicians). Twenty five (89\%) (Fig. 3b) used oral prednisolone as part of TED treatment; periocular steroids were used by ten (36\%) respondents (Fig. 3c); 26 (93\%) offered second-line immunosuppression within their own unit (Fig. 3d) and 26 (93\%) used orbital radiotherapy-18 within own trust and eight referral to another unit and not used by two respondents as part of TED treatment (Fig. 3e).

The text of both Surveys 1 and 2 are available in Supplementary Fig. 1.

The referral pattern and local availability of TED treatments are detailed in Supplementary Fig. 2.

\section{Discussion}

The concept of combined multidisciplinary clinics and networks has now been well established in many disciplines, most notably in oncology where it works best to harness all available expertise to optimise patient management and outcomes. It has also shown that it improves the time to diagnosis and treatment in TED [16]. We recognise that to formulate recommendations, there has to be a process of consensus and its impact would be greater if embraced by multiple professional organisations, especially when the topic is such that it transcends more than one specialty.

This nationwide survey of ophthalmologists with an interest in TED, being voluntary, represents a valuable 'snapshot' of provision rather than a complete inventory of TED care in the UK. The study was deliberately designed as a two stage with a short Survey 1 to encourage participation with more brief and general questions and Survey 2 to elicit more information from those presumably already running TED specialist clinics. With a $41 \%$ response to Survey 1 rate if we assume that the responders are the 'enthusiasts', then the data suggest that care for TED is not provided in a multidisciplinary context with simultaneous endocrinology and ophthalmology input in up to $40 \%$ of ophthalmology clinics and the presence of geographical clusters leading to uneven access to healthcare.

Whilst there is undoubtedly a desire for many endocrinology specialists to work within a multidisciplinary setting, as demonstrated by a recent 'snap shot' survey of endocrinologists [17], there is significant variation in clinical practice. The reasons for lack of multidisciplinary sessions are likely multifold. Firstly, many regions of the UK 
may be spread across large geographical areas, particularly when endocrinology and ophthalmology are not located on the same site. Furthermore, provisions in the workforce may limit the desire to take on additional clinics to support multidisciplinary working. We speculate that much of this perception of lack of support is due to lack of clear lines of communication and may be hampered by the geographical separation in some regions.

A few areas of the country appear not to be using intravenous steroids and there is high usage of oral steroids despite evidence for better efficacy and safety with intravenous steroids. However, given the way that the questionnaire was phrased the aim was to ascertain the different treatment modalities in use in the UK rather than the choice of first-line treatment. The recent BOSU national wide surveillance on dysthyroid optic neuropathy found that $82 \%$ of reporting units followed the EUGOGO protocol for severe eye disease so this would suggest that many units in the UK do follow the EUGOGO recommendations. The significant use of oral steroids (89\%), may reflect its use as part of bridging treatment for second-line immunosuppression, where the 'safe upper limit' of intravenous steroid dose of $8 \mathrm{~g}$ had been exceeded or as cover for radioiodine treatment to mitigate the risk of orbitopathy progression.

There is also relative high use of periocular steroids $(36 \%)$ despite lack of comparative efficacy and safety data on this mode of administration compared with systemic steroids. Again this may not be the respondent's first-line treatment of choice but utilised as adjunctive treatment to intravenous steroids or where the first-line treatment of choice is medically contraindicated, e.g. uncontrolled diabetes or in an already immunosuppressed patient or where severe complications necessitated the termination of the course of treatment $[18,19]$. It is of note that even among this group of possible 'enthusiasts' second-line medical therapy after steroids was not used by $32 \%$ of respondents and in many areas was provided in a different trust. This is particularly relevant in view of recent evidence of the marginal benefits of adjunctive therapy combined with steroids $[20,21]$. Access to orbital decompression surgery seems available in most parts of the UK, though frequently in a different trust.

It is also apparent that the process of actively screening for TED is suboptimal and not standardised and important outcome data such as quality of life measures are not routinely collected. Multidisciplinary relationships between ophthalmology and endocrinology teams could also be improved in many centres. The importance of early diagnosis and treatment of TED for a better outcome is clearly underlined by the Amsterdam declaration [2]. Results from our study suggest that although progress is being made to achieve these goals, there is still a substantial need for further improvement. Optimal disease management of TED can span across many different specialties (Supplementary Fig. 3) and treatment timing is crucial to maximise efficacy of the treatments in the acute phase, including thyroid hormonal control and control of the orbital inflammation [22].

Limitations of our BOPSS survey include that there may be more than one respondent from the same unit completing the survey. Despite multiple invitations and extensive consultation at various national meetings over half of the BOPSS full membership did not respond to the survey. Moreover, respondents are also more likely to be a selfselecting cohort with specialist interest in TED. It is therefore likely that they are overall better informed on TED, and thus reality is likely under-represented with greater variation and even less access to multidisciplinary working than described in our study.

We believe two major approaches are required to achieve fuller implementation and better uptake of the Amsterdam Declaration. Firstly, the RCP guidance requires further reinforcement and dissemination with adoption by all clinical units, as part of adoption of the TEAMeD-5 programme, and secondly, units treating TED should be able to demonstrate compliance with auditable benchmarks.

While recognising the UK environment of finite resources within a national health service BOPSS drafted general standards, which we believe should be achievable within current resources (Table 1). This predominantly requires improved communication between ophthalmologists and endocrinologists and the establishment of locally agreed operating procedures taking into account existing service configurations.

After extensive consultation with BOPSS members through discussion groups and presentations at executive meetings, as well as the annual general meeting, the consensus was that there should be evidence based national benchmarks. However, implementation policies were felt by the membership best decided by local stakeholders taking into account variation in service configurations and variation across the country.

The proposed benchmarks (Table 1) received broad acceptance across the BOPSS membership and the TEAMeD stakeholder representatives. An initiative to allocate a section of the members website to allow for uploading of local practice guidelines to enable sharing of best practice also received executive endorsement. In addition, the BOPSS executive committee fully supported the recent TEAMeD-5 proposal of identifying a lead endocrinologist for each specific region to take ownership and coordinate implementation of the programme. The role should entail providing specialist training in diagnosis and management of TED. Furthermore, it would involve ensuring referrals are managed in a timely fashion, with a proposed pathway to establish joint endo-ophthalmology 
Table 1 Proposed standards of care for multidisciplinary working between ophthalmologists and endocrinologists

To be agreed with referring endocrinologists

\begin{tabular}{|c|c|}
\hline Essential & Desirable \\
\hline SOP for screening and referral & Validated screening tool with audit of outcomes \\
\hline Increased awareness of thyroid eye disease among all Graves' disease patients & TEAMeD thyroid eye disease early warning card \\
\hline Smoking cessation & Sign post and refer to smoking cessation services \\
\hline Initiation of dry eye treatment as necessary in endocrine clinic & Locally agreed treatment protocol \\
\hline Clear guidelines regarding suitability for radioiodine treatment & Local SOP for thyroid eye clinic referral \\
\hline Clear routes of Communication & MDT clinic \\
\hline \multicolumn{2}{|l|}{ Eye clinic standard operating procedures (SOP) } \\
\hline \multirow[t]{2}{*}{ Measures to avoid loss of follow up of patients with active orbitopathy } & Fail-safe officer \\
\hline & GDPR compliant patient database \\
\hline Comprehensive clinic assessment & $\begin{array}{l}\text { Dedicated clinic protocol, regular QOL assessment and } \\
\text { clinical photographs }\end{array}$ \\
\hline \multirow{2}{*}{$\begin{array}{l}\text { Orthoptic assessment for all patients with diplopia at baseline and at regular } \\
\text { intervals to monitor progression/ treatment effect }\end{array}$} & Local SOP \\
\hline & Recommendations include Hess and BSV assessments \\
\hline Local agreed SOP for use of orbital imaging & MDT radiology meeting \\
\hline Written patient information & $\begin{array}{l}\text { Standard package of information leaflets covering all } \\
\text { aspects of thyroid eye disease }\end{array}$ \\
\hline SOP for immunosuppression (first and second line) & Locally agreed treatment and referral protocol \\
\hline SOP for orbital radiotherapy (if used) & Locally agreed treatment and referral protocol \\
\hline SOP for sight-threatening disease & Locally agreed treatment and referral protocol \\
\hline SOP for rehabilitation treatment & Locally agreed treatment and referral protocol \\
\hline
\end{tabular}

SOP standard operating procedure

services, and to include an audit programme, detailing surgical activity and treatment outcomes. The standards set should include 'referral for assessment for TED' and use of a validated screening tool (e.g. the Vancouver orbitopathy score or the DiaGO) [23, 24]. As demonstrated in our survey, there is significant lack of clarity how patients are identified for referral.

We concur with TEAMeD-5 that all treating doctors should alert patients about risk of TED [15]. Best practice would be to use the TEAMeD TED early warning card [15] to help identify patients with moderate/severe and sightthreatening disease. It is well established that radioiodine can exacerbate the onset and progression of TED [25], therefore it is crucial that the two specialties agree to reduce this risk particularly in smokers. All treating doctors should offer smoking cessation advice and recommend referral to smoking cessation services to all TED/Graves' patients who are smokers. A previous prospective multi-centre study in the UK highlighted that not enough patients are being provided with smoking cessation advice and information on the impact of smoking on TED and control of thyroid function [11]. Moreover, quality of life indicators could routinely be incorporated into the standard consultation template [26-28]. More clearly sign posted access to psychological support would likely be beneficial.
The BOPSS executive committee has supported allocating a dedicated website area so that members can contribute their local standard operating procedures (SOPs) to enable sharing of best practice. Locally agreed SOP should include active screening for TED, indications for steroid cover for Graves' patients undergoing radioiodine treatment, and protocols for second-line immunosuppression. Local SOP should additionally incorporate the use of immunosuppression orbital radiotherapy and follow up protocol, as well as planning for rehabilitative treatment. In view of many patient related adverse events related to clinical administration, an SOP should be developed to prevent patients with active thyroid orbitopathy from being lost to follow up and to be seen at suitable intervals. Examples of best practice would include a secure GDPR compliant TED patient database and a 'fail-safe officer'/multidisciplinary coordinator. This is in line with the RCP recommendations as TED is a condition with increased risk [29]. We recommend all units treating TED should have an agreed protocol with referring endocrinologists as to the thyroid treatment and monitoring with clear documentation of communication and treatment with best practice having a specialist joint thyroid eye clinic with an endocrinologist and ophthalmologist in the same clinic. 
Table 2 Proposed audit criteria for review of services managing thyroid eye disease
(1) Efficacy

Consideration of oral selenium supplements $(91.3 \mathrm{mcg}$ elemental selenium bd for 6 months) for patients with mild, active TED.

Smoking cessation advice for patients who are smokers (Target $80 \%$ ).

Prompt correction of dysthyroidism and maintenance of euthyroidism.

Where systemic steroid treatment is indicated, use of intravenous pulses of methylpred-nisolone in preference to oral steroids.

Availability of urgent treatment for sight-threatening orbitopathy, including surgical decompression for patients who fail to respond to high dose intravenous steroids.

Availability of orbital irradiation.

Availability of rehabilitative surgery for patients with inactive or minimally active disease who are significantly impaired, socially or psychologically as a result of TED.

(2) Safety Appropriate selection of patients with TED who are being considered for radioiodine for suitability of steroid cover.

Safe use of immunosuppressive treatments (exclusion of those for whom there are contraindications, assessment and monitoring of risks of serious adverse effects.

Timely assessment of response to high dose intravenous steroids and withdrawal of steroid treatment in favour of other therapies for those with inadequate response.

(3) Patient-centred care

(4) Timely

(5) Efficient

(6) Equitable
Availability of good quality information about TED, its usual course, likely outcomes and potential treatments, complemented by high quality written information and access to patient-led organisations.

Formulation of personalized management plans following multidisciplinary discussion.

Good communication between the clinical team and the patient.

Patient engagement with the decision process about management of TED.

Use of validated tools to assess the impact of TED on their quality of life, like the GO-QOL.

Patients with sight-threatening TED (dysthyroid optic neuropathy resulting in significant reduction in visual acuity, corneal breakdown with impending or established infection, globe subluxation) should be treated urgently within 2 weeks.

Patients with moderately to severe, active TED should be offered treatment within a six weeks from presentation.

Multiple surgical treatments in patients requiring complex rehabilitative surgery, should follow the sequence: orbital decompression/eye muscle surgery/lid surgery.

Referral pathways from primary to secondary and tertiary care, should be well defined and seamless.

All patients should have access to excellent treatment.
Within NHS trusts, audit should include numbers of patients treated in each of the above categories and also the most important metric-time from onset of symptoms of eye disease to first treatment. It would not be controversial to recommend that treatment for acute visual loss should be initiated urgently.

To summarise, Table 1 outlines the recommended standards for multidisciplinary working between ophthalmologists and endocrinologists. Table 2 summarises the combined quality standards for management of TED. In conclusion, our data suggest that management of TED across the UK is varied, with clinical practice not reflecting national and regional guidelines. Evolution of local policies and multidisciplinary teams are critical to address this deficiency under the auspices of the TEAMeD-5 (Supplementary Figs. 4) initiative. Hopefully a devolved approach through identifying lead endocrinologists in each region to take ownership and coordinate implementation of the TEAMeD-5 programme will allow a structured training program to fellow clinicians to ensure that an appropriately configured and expert multidisciplinary service for managing moderate-to-severe cases of TED is present in all regions.

\section{Summary}

\section{What was known before}

- The TEAMeD-5 programme recommends all patients with moderate-to-severe TED should have access to multidisciplinary clinics with combined Ophthalmology and Endocrinology expertise 


\section{What this study adds}

- This nationwide survey of ophthalmologists with an interest in TED represents a valuable snapshot of provisions of TED care in the UK, with findings suggestive of scope for improvement.

- In this paper, we recommend a framework and recommendations for more robust collaboration across specialties and propose standards endorsed by multidisciplinary stakeholder societies.

Acknowledgements The recommendations in this paper have been endorsed by TEAMeD, BOPSS, Royal College of Ophthalmologists, The Royal College of Physicians, Society for Endocrinology, British Thyroid Foundation and the British and Irish Orthoptic Society. The authors would like to thank the BOPSS Thyroid Audit Standards Group: Vikas Chadha, Lucy Clarke, Rebecca Ford, Anjana Haridas, Carole Jones (BOPSS president 2018), Faye Mellington, Rachna Murthy, Jonathan Norris, Cornelius Rene (BOPSS president 2016-2018), Sachin Salvi, Jimmy Uddin, Colin Vize (BOPSS secretary) and Stephen White and the BOPSS membership for completing the surveys. The authors also thank Janis Hickey (British Thyroid Foundation) and Lorna Pankethman (British and Irish Orthoptic Society) for their support and recommendations. The authors would like to thank TEDct for their generous donation that made it possible for this paper to be open access.

\section{Compliance with ethical standards}

Conflict of interest Ben Williams is the Webmaster for BOPSS and is renumerated for providing IT support. None of the other authors or contributors have any conflict of interest to declare.

Publisher's note Springer Nature remains neutral with regard to jurisdictional claims in published maps and institutional affiliations.

Open Access This article is licensed under a Creative Commons Attribution 4.0 International License, which permits use, sharing, adaptation, distribution and reproduction in any medium or format, as long as you give appropriate credit to the original author(s) and the source, provide a link to the Creative Commons license, and indicate if changes were made. The images or other third party material in this article are included in the article's Creative Commons license, unless indicated otherwise in a credit line to the material. If material is not included in the article's Creative Commons license and your intended use is not permitted by statutory regulation or exceeds the permitted use, you will need to obtain permission directly from the copyright holder. To view a copy of this license, visit http://creativecommons. org/licenses/by/4.0/.

\section{References}

1. Abraham-Nordling M, Byström K, Törring O, Lantz M, Berg G, Calissendorff $\mathrm{J}$, et al. Incidence of hyperthyroidism in Sweden. Eur J Endocrinol. 2011;165:899-905.

2. Perros P, Hegedus L, Bartalena L, Marcocci C, Kahaly GJ, Baldeschi L, et al. Graves' orbitopathy as a rare disease in Europe: a European Group on Graves' Orbitopathy (EUGOGO) position statement. Orphanet J Rare Dis. 2017;12:72.
3. Ponto KA, Merkesdal S, Hommel G, Pitz S, Pfeiffer N, Kahaly GJ. Public health relevance of Graves' orbitopathy. J Clin Endocrinol Metab. 2013;98:145-52.

4. Park JJ, Sullivan TJ, Mortimer RH, Wagenaar M, Perry-Keene DA. Assessing quality of life in Australian patients with Graves' ophthalmopathy. Br J Ophthalmol. 2004;88:75-8. http://www. ncbi.nlm.nih.gov/pubmed/14693779.

5. Marcocci C, Altea MA, Leo M. Treatment options for Graves' orbitopathy. Expert Opin Pharmacother. 2012;13:795-806.

6. Perros P, Wiersinga WM. The Amsterdam Declaration on Graves' orbitopathy. Thyroid. 2010;20:245-6.

7. Perros P, Dayan CM, Dickinson AJ, Ezra D, Estcourt S, Foley P, et al. Management of patients with Graves' orbitopathy: initial assessment, management outside specialised centres and referral pathways. Clin Med. 2015;15:173-8.

8. Bartalena L, Baldeschi L, Dickinson A, Eckstein A, KendallTaylor P, Marcocci C, et al. Consensus statement of the European Group on Graves' orbitopathy (EUGOGO) on management of GO. Eur J Endocrinol. 2008;158:273-85.

9. Bartalena L, Baldeschi L, Boboridis K, Eckstein A, Kahaly GJ, Marcocci C, et al. The 2016 European Thyroid Association/European Group on Graves' Orbitopathy Guidelines for the Management of Graves' Orbitopathy. Eur Thyroid J. 2016;5:9-26.

10. Estcourt S, Hickey J, Perros P, Dayan C, Vaidya B. The patient experience of services for thyroid eye disease in the United Kingdom: results of a nationwide survey. Eur $\mathrm{J}$ Endocrinol. 2009;161:483-7.

11. Mellington FE, Dayan CM, Dickinson AJ, Hickey JL, MacEwen $\mathrm{CJ}$, McLaren J, et al. Management of thyroid eye disease in the United Kingdom: a multi-centre thyroid eye disease audit. Orbit. 2017;36:159-69.

12. Perros P, Baldeschi L, Boboridis K, Dickinson AJ, Hullo A, Kahaly GJ, et al. A questionnaire survey on the management of Graves' orbitopathy in Europe. Eur J Endocrinol. 2006;155:207-11.

13. Prummel MF, Bakker A, Wiersinga WM, Baldeschi L, Mourits MP, Kendall-Taylor P, et al. Multi-center study on the characteristics and treatment strategies of patients with Graves' orbitopathy: the first European Group on Graves' Orbitopathy experience. Eur J Endocrinol. 2003;148:491-5.

14. Perros P, Chandler T, Dayan CM, Dickinson AJ, Foley P, Hickey $\mathrm{J}$, et al. Orbital decompression for Graves' orbitopathy in England. Eye. 2012;26:434-7.

15. Draman MS, Mitchell A, Dayan C. TEAMeD-5: improving outcomes in thyoid eye disease I Society for Endocrinology. Endocrinologist. 2017;(125). https://www.endocrinology.org/endocrinologist/ 125-autumn17/features/teamed-5-improving-outcomes-in-thyoid-eyedisease/.

16. Benzimra JD, Quinn AG, Kersey T, McGrane D, Goss L, Vaidya B. Management of patients in a combined thyroid eye clinic in secondary care. Int Ophthalmol. 2014;34:1-6.

17. Avari C, Bravis V, Robinson S, Meeran K, Lee V. Sharing and caring? Perspectives of multidisciplinary working between endocrinologists and ophthalmologists in thyroid eye disease I Society for Endocrinology. Endocrinologist. 2018;(129). https://www. endocrinology.org/endocrinologist/129-autumn18/general-news/ sharing-and-caring-perspectives-of-multidisciplinary-workingbetween-endocrinologists-and-ophthalmologists-in-thyroid-eyedisease/.

18. Ebner R, Devoto MH, Weil D, Bordaberry M, Mir C, Martinez H, et al. Treatment of thyroid associated ophthalmopathy with periocular injections of triamcinolone. Br J Ophthalmol. 2004;88:1380-6.

19. Young SM, Kim YD, Lang SS, Woo KI. Transconjunctival triamcinolone injection for upper lid retraction in thyroid eye disease-a new injection method. Ophthal Plast Reconstr Surg. 2018;34:587-93. 
20. Rajendram R, Taylor PN, Wilson VJ, Harris N, Morris OC, Tomlinson $\mathrm{M}$, et al. Combined immunosuppression and radiotherapy in thyroid eye disease (CIRTED): a multicentre, $2 \times 2$ factorial, double-blind, randomised controlled trial. Lancet Diabetes Endocrinol. 2018;6:299-309.

21. Kahaly GJ, Riedl M, Konig J, Pitz S, Ponto K, Diana T, et al. Mycophenolate plus methylprednisolone versus methylprednisolone alone in active, moderate-to-severe Graves' orbitopathy (MINGO): a randomised, observer-masked, multicentre trial. Lancet Diabetes Endocrinol. 2018;6:287-98.

22. Baldeschi L, Wakelkamp IM, Lindeboom R, Prummel MF, Wiersinga WM. Early versus late orbital decompression in Graves' orbitopathy: a retrospective study in 125 patients. Ophthalmology. 2006;113:874-8.

23. Mitchell AL, Goss L, Mathiopoulou L, Morris M, Vaidya B, Dickinson AJ, et al. Diagnosis of Graves' orbitopathy (DiaGO): results of a pilot study to assess the utility of an office tool for practicing endocrinologists. J Clin Endocrinol Metab. 2015;100: E458-62.
24. Dolman PJ. Evaluating Graves' orbitopathy. Best Pr Res Clin Endocrinol Metab. 2012;26:229-48.

25. Vannucchi G, Campi I, Covelli D, Dazzi D, Curro N, Simonetta S, et al. Graves' orbitopathy activation after radioactive iodine therapy with and without steroid prophylaxis. J Clin Endocrinol Metab. 2009;94:3381-6.

26. Wickwar S, McBain HB, Ezra DG, Hirani SP, Rose GE, Newman SP. What are the psychosocial outcomes of treatment for thyroid eye disease? A systematic review. Thyroid. 2014;24:1407-18.

27. Estcourt S, Quinn AG, Vaidya B. Quality of life in thyroid eye disease: impact of quality of care. Eur $\mathrm{J}$ Endocrinol. 2011;164:649-55.

28. Wickwar S, McBain H, Ezra DG, Hirani SP, Rose GE, Newman SP. The psychosocial and clinical outcomes of orbital decompression surgery for thyroid eye disease and predictors of change in quality of life. Ophthalmology. 2015;122:2568-76.e1

29. The Royal College of Ophthalmologists. New Guidelines for Thyroid Eye Disease. 2015. https://www.rcophth.ac.uk/2015/07/ new-guidelines-for-thyroid-eye-disease/. 